\title{
A Simple and Interpretable Predictive Model for Healthcare
}

\author{
Subhadip Maji ${ }^{\dagger}, *$, Raghav Bali ${ }^{\dagger}, *$, Sree Harsha Ankem ${ }^{\dagger}$, Kishore V Ayyadevara ${ }^{\dagger}$ \\ † Optum Global Solutions, India
}

\begin{abstract}
Deep Learning based models are currently dominating most state-of-the-art solutions for disease prediction. Existing works employ RNNs along with multiple levels of attention mechanisms to provide interpretability. These deep learning models, with trainable parameters running into millions, require huge amounts of compute and data to train \& deploy and adversely impact real world usage. We address these challenges by developing a simpler yet interpretable tree based model. We model and showcase results on the task of predicting first occurrence of a diagnosis, often overlooked in existing works. We push the capabilities of a tree based model and come up with a strong baseline for more sophisticated models. Our work shows an improvement over deep learning based solutions all the while maintaining interpretability.
\end{abstract}

Keywords: Healthcare, Disease Prediction, Boosted Trees, Deep Learning, EHR

\section{Introduction}

Deep Learning has become the go to choice for developing solutions in areas such as image processing, text processing, and even healthcare. Usage of Recurrent Neural Networks (RNNs) to model sequential data has been seen in many recent works[1, 2] . Advancements in deep learning space through the use of attention mechanisms[3] has empowered such black-box models with interpretability. RNNs with Attention in healthcare have also been successfully applied in several works[4, 5]. Choi, Bahadori, Sun, Kulas, Schuetz, and Stewart [6] used reversed time attention mechanism to achieve good performance while being clinically interpretable for application to the Electronic Health Records (EHR) data. Ma, Chitta, Zhou, You, Sun, and Gao [7] rectified some drawbacks of RETAIN, by introducing bidirectional RNNs to capture both the past and future medical experiences of patients. Several works have highlighted the positive impact of such predictive models towards improving quality of healthcare [8]. This boost in performance of deep learning models comes at the cost of compute requirements, training time and volume of training data. In many cases, these costs are prohibitory both financially and otherwise.

We address these challenges by proposing a simpler yet interpretable tree based predictive model for healthcare. The following were the major motivations behind this work. First and foremost was to develop a competitive baseline which can act as a benchmark for highly parameterised deep learning approaches. Second was to provide a novel way of preparing sequential EHR data for non-deep learning approaches. Our approach had significant impact on overall model performance. The final motivation was to provide an efficient and easy to deploy alternative to deep learning models while providing on-par performance and interpretability.

Our model was tested on multiple internal EHR datasets, each having at least a 24 month historical timeline. We experimented with different datasets and target diseases to ensure generalizable performance. We also cater to first-occurrence prediction, i.e. predicting the first ever incidence of a diagnosis in consideration. This constraint adds additional complexity to the prediction task. Our experiments showcase that our simpler approach improves over deep learning based solutions all the while maintaining interpretability.

*subhadipmaji.jumech@gmail.com, raghavbali@optum.com

This article is () 2021 by author(s) as listed above. The article is licensed under a Creative Commons Attribution (CC BY 4.0) International license (https://creativecommons.org/licenses/by/4.0/legalcode), except where otherwise indicated with respect to particular material included in the article. The article should be attributed to the author(s) identified above. 
Table 1. Summary of EHR Datasets utilised for experiments

\begin{tabular}{|l|c|c|c|}
\hline & Diabetes & Heart Failure & Kidney Failure \\
\hline \# of patients & 261,316 & 84,878 & 85,556 \\
\hline \# of medical code groups & 1,840 & 3,327 & 3,327 \\
\hline Avg. \# of medical codes per patient & 125.84 & 69.76 & 73.89 \\
\hline Incidence Rate & $12 \%$ & $15 \%$ & $14 \%$ \\
\hline
\end{tabular}

The rest of the paper is organized as follows: section 2 details the data preparation step. It had a significant impact on the overall model performance. Section 3 describes the overall approach, model choice along with different experiments and their results. In section 4 we discuss the need for model interpretability. We also showcase instance level interpretability results using a model agnostic approach called SHAP[9]. Section 5 presents commentary on the effectiveness of our work in this domain and section 6 concludes the paper.

\section{Data Preparation}

The aim is to prepare longitudinal EHR data for first occurrence prediction task. To capture enough historical traits and variability, similar to [6], we use a 24 month historical period for training. For the predictions to be useful and actionable, we use a delta of 3 months between the training period and first occurrence date of the diagnosis. Table 1 showcases a quick summary of our EHR dataset.

Let us denote a patient as $p$ having a certain history of diagnosis, denoted as $H=$ $\left\{t_{1}, t_{2}, \ldots . t_{N}\right\}$ where $t_{i}$ is one timestep or a visit in his/her history. Each timestep consists of various clinical codes such as diagnosis codes (ICD codes), procedures codes (CPT codes), prescription (RX codes) and demographic details of the patient which can be represented as $t_{i}=\left\{I C D_{(1 \ldots N)}, C P T_{(1 . . N)}\right.$, demographics $\}$. Assume that the task is to predict the first occurrence of a disease $D$. Given that this patient was diagnosed with $D$ at time steps $t_{n}$ and $t_{(n+k)}$ (where $k>0$ ) time step. The first occurrence of $D$ for $p$ is the time step $t_{n}$ which would be our target instance. The response variable $i s \_D$ (for instance $i s \_d i a b e t i c$ ) would be set to 1 for such patients and 0 otherwise (i.e. patients who have never been diagnosed with diabetes). The feature space consists of $I C D \_$codes along with demographic attributes like age and gender. To prepare an aggregated feature vector for each patient $p$, we dissolve the time steps, i.e. the patient vector is represented as:

$$
p=\left\{I C D_{1} . . I C D_{N}, C P T_{1}, . . C P T_{N}, R X_{1} . . R X_{N}, \text { age }, \text { gender }\right\},
$$

where the value for each $I C D_{i}, C P T_{i}$ and $R X_{i}$ is set to the number of such diagnosis, procedures or medication respectively. So, for a given patient $p_{i}$ the feature vector would be referred as:

$$
p_{i}=\left\{X_{i}, y_{i}\right\}:\left\{\left(n_{11}, n_{12}, \ldots . ., a_{1}, g_{1}, \ldots\right), y_{i}\right\},
$$

where $n_{i j}$ is the ICD count value for patient $i$ and ICD $j$ (similarly for CPT and RX codes), $a_{i}, g_{i}$ and $y_{i}$ are the age, gender and response value respectively for patient $i$; with $a_{i} \in[0, \infty), g_{i} \in\{$ Male, Female $\}$ and $y_{i} \in\{0,1\}$.

\section{Experimental Setup}

We chose tree-based model, XGBoost[10] for this task because of their low bias, robustness against outliers, ease of interpretability and speed of training and inference. We explored the use of model-agnostic instance level feature interpreter SHAP [9] as well. Figure 1 shows our overall approach in a flow diagram for reference. 


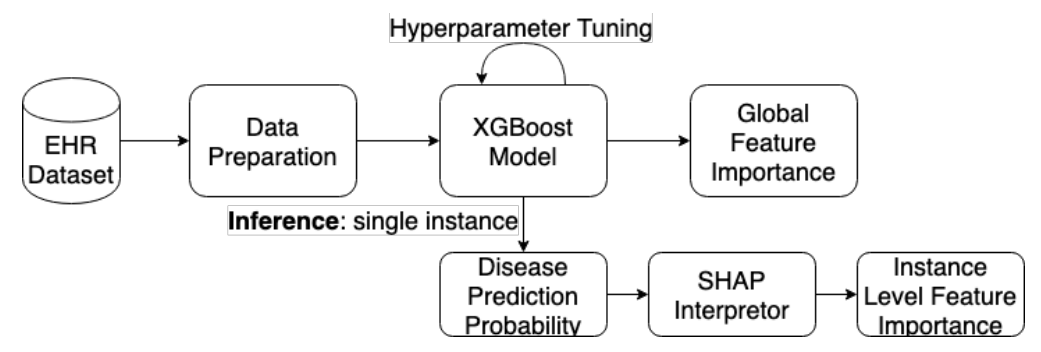

Figure 1. Flow Diagram of the overall approach for rare disease prediction

As our datasets are imbalanced, we chose Area Under Receiver Operating Characteristic Curve or AUROC[11] as our metric of choice. We also measure Recall@K as an additional metric and define it as: given the predicted probability scores across all the observations binned into deciles. Thus, Recall@30 is defined as the percentage of true cases for which the predicted probability falls in the top 3 deciles.

\subsection{Experiments and Results}

As a baseline model, we fit a logistic regression on our datasets and it provides test AUROC of 0.711, 0.754 and 0.731 for diabetes, heart failure and kidney failure respectively. For the rest of this section we will refer to count based feature set as our primary dataset. We mark datasets with a subscript oneHot where we make use of binary categorized features.

The next experiment involved fitting an XGBoost model with default parameters, $x g b_{d}^{D}$, where $d$ denotes default parameters and $D$ denotes the disease for which the model was trained. We will use the subscript $o$ to denote the optimized/fine-tuned model. The $x g b_{d}^{D}$ models for diabetes, heart failure and kidney failure resulted in test AUROC values of 0.78 , 0.837 and 0.823 respectively. This is good improvement over the logistic regression baseline.

We performed greedy search based hyper-parameter tuning to try and push the boundaries for fine-tuning. The fine-tuned XGBoost (example $x g b_{o}^{\text {diabetes }}$ ) shows an improvement of approximately $6.5 \%$ over $x g b_{d}^{\text {diabetes }}$ with an AUROC of 0.8281 for diabetes. We observed similar lifts for heart failure and kidney failure models as well with corresponding AUROC at 0.853 and 0.849 respectively.

\subsection{Comparison with Recent works}

Works $[6,7]$ compare their results against weak baselines such as logistic regression only and overlook the task of first occurrence prediction. We believe this additional constraint is an important one for the models to be useful in real life use cases. We also observed that the performance (across models) tends to improve drastically if this constraint is removed. This makes intuitive sense as for many diagnosis, a repeat occurrence is quite obvious. From a business and healthcare stand-point, it makes sense to predict first occurrence in order to perform any preventive/corrective action in time.

To provide a common framework, competitive baseline and useful constraints, we trained RETAIN[6] and Dipole[7] along with hyperparameter tuning on our datasets. We use the same train-validation-test splits for each of the diseases for RETAIN and Dipole. We prepared training data in the format expected by these works and performed hyper-parameter tuning to report the best results on our test dataset. Upon comparison, the surprising aspect was the comparison with our fine-tuned XGBoost model, i.e. $x g b_{o}$ shows considerable improvements as compared to RETAIN and Dipole for all three target diseases. The results are showcased in Table 2 for reference. 
Table 2. Experiment Results for different models based on described ICD3 feature sets

\begin{tabular}{|l|l|l|l|l|l|l|}
\hline & \multicolumn{2}{|c|}{ Diabetes } & \multicolumn{2}{c|}{ Kidney Failure } & \multicolumn{2}{c|}{ Heart Failure } \\
\hline Model & \multicolumn{1}{|c|}{ AUROC } & Recall@30 & AUROC & Recall@30 & AUROC & Recall@30 \\
\hline RETAIN & $0.7831 \pm 0.0062$ & 0.2998 & $0.8202 \pm 0.001$ & 0.3312 & $0.8385 \pm 0.002$ & 0.4047 \\
\hline Dipole & $0.7901 \pm 0.0085$ & 0.3002 & $0.8211 \pm 0.008$ & 0.3357 & $0.8291 \pm 0.009$ & 0.3998 \\
\hline $\begin{array}{l}\text { Logistic } \\
\text { Regression }\end{array}$ & $0.7113 \pm 0.0000$ & 0.2885 & $0.7319 \pm 0.0000$ & 0.3129 & $0.7543 \pm 0.0000$ & 0.3765 \\
\hline$x g b_{d}$ & $0.78 \pm 0.000$ & 0.3047 & $0.8233 \pm 0.0001$ & 0.3301 & $0.8377 \pm 0.0002$ & 0.3975 \\
\hline$x g b_{\text {one }}$ ot & $0.805 \pm 0.0003$ & 0.3052 & $0.8258 \pm 0.0004$ & 0.3311 & $0.8404 \pm 0.0000$ & 0.4002 \\
\hline xgb $_{\mathbf{o}}$ & $\mathbf{0 . 8 2 8 1} \pm \mathbf{0 . 0 0 0 5}$ & $\mathbf{0 . 3 0 9 3}$ & $\mathbf{0 . 8 4 9 7} \pm \mathbf{0 . 0 0 0 7}$ & $\mathbf{0 . 3 3 7 4}$ & $\mathbf{0 . 8 5 3 1} \pm \mathbf{0 . 0 0 0 5}$ & $\mathbf{0 . 4 1 1 3}$ \\
\hline
\end{tabular}

The experiments and results outlined in this section utilised ICD codes truncated till 3 characters (or ICD3 for short). For instance, diagnosis code 250.31 refers to Diabetes with other coma, type I [juvenile type], not stated as uncontrolled. We truncate 250.31 to only 250 which refers to the class of diabetic diagnosis. By doing so, we reduce the overall dimensionality of our already sparse feature set.

\subsection{Experiments with Full ICD Feature Set}

The ICD3-grouping is helpful in reducing the impact of a sparse feature set, yet it leads to loss of understanding/interpretability as well as information. To enable better and granular interpretation, in this section, we experimented with complete ICD codes or ICD-Full. The dataset was prepared as mentioned in the section 2 with the only difference being that the new feature set consists of ICD-Full while targets are still ICD3. This was done to ensure we have enough training samples for each class. This new dataset was used to train and tune RETAIN, Dipole and XgBoost for comparison. Similar to previous experiments, in this case as well XGBoost outperformed its more sophisticated competitors on AUROC metric. Results are shared in table 2 for reference. We attribute the improvement in performance across models to the added granularity in the feature set all the while maintaining similar class distribution. The results were cross validated to ensure model stability. We achieved test AUROC scores of $0.8626 \pm 0.007,0.8539 \pm 0.001$ and $0.8561 \pm 0.02$ for heat-failure prediction using $x g b_{o}$, RETAIN and Dipole respectively.

\section{Interpretability}

The XGBClassifier[12] is interprerable at a global level as it provides functionality for identification of important features out of the box. Important features for $x g b_{o}$ for first occurrence prediction of diabetes are reported as ICD_I10(Hypertension), ICD_R73(Elevated blood glucose levels), etc. which are inline with factors leading to diabetes (this is medically validated). Heart Failure task using ICD-Full as feature set resulted in top features as ICD5939 (Unspecified disorder of kidney and ureter) and ICD7931 (Nonspecific (abnormal) findings on radiological and other examination of lung field).

For instance-level interpretability, let us work through an instance of a patient from our test dataset for diabetes. We use $x g b_{o}$ to predict the first occurrence probability of this patient being diabetic as 0.979 (ground truth for this patient was observed to be 1). Upon analysing this instance using SHAP, we observe the following for this particular patient. Features such as age, LAB_4548-4_H (a diagnostic test for Haemoglobin A1c), RX_841(diabetes testing supplies), etc. have positive SHAP values. These are the past diagnosis, events, lab test or prescriptions which the model uses to predict with a high probability score (0.979). The same is visually showcased for heart failure and kidney failure as well in figure 2 for reference. Similar to attention based interpretation plots as showcased in 


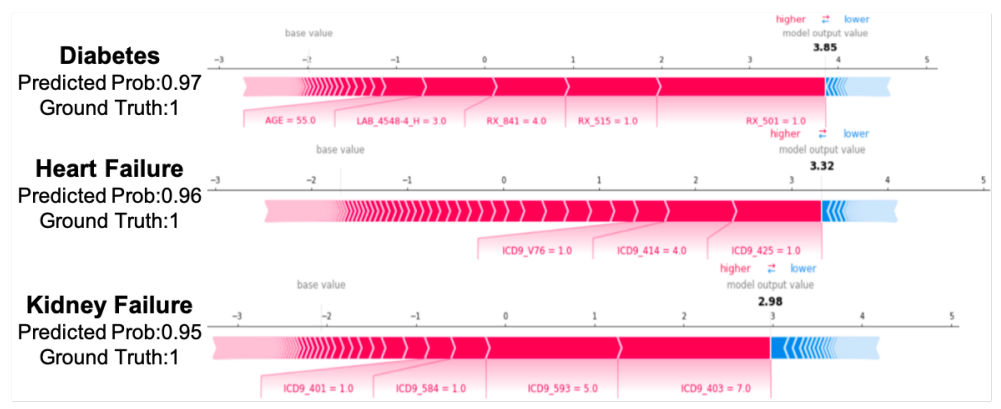

Figure 2. Interpreting Patient Level Predictions using SHAP for 3 randomly chosen patients for diabetes, heart failure and kidney failure prediction tasks. The plots showcase logit-value for each of the contributing features.

RETAIN[6], we leverage SHAP values and force plots to provide patient level interpretability for our work.

\section{Effectiveness of Tree Based Approaches}

The importance of sequential/longitudinal nature of EHRs is paramount and the chronological sequence of events is an important factor in a number of diagnosis (such as Alzheimer's ). Yet, not all diagnosis are time dependent, for instance, the target diseases in our experiments. Our consultations with medical experts highlighted the fact that even though past diagnosis impact current and future health states, the time gap is not always an important factor. This goes hand in hand with our results and the fact that a simpler model out performs complex ones.

Despite successful application across various domains and data types (mostly unstructured), deep learning is yet to make a mark when it comes to tabular or structured datasets. Tree based ensembles, especially XGBoost and variants dominate this space[13]. Real world datasets are typically high dimensional but the feature sets are sparse in nature. In other words, they can be represented in a lower dimensional space easily (say a hyperplane). This process is termed as unfolding or manifold learning. Tree based boosting algorithms are highly efficient for manifold learning with hyperplane boundaries (a characteristic of tabular datasets) [14]. Deep Learning models have millions of parameteris and even though they are termed as universal function approximators, finding the optimal set of parameters is non-trivial. These require far more training samples and time as compared to traditional methods[15].

\section{Conclusion}

We presented a simple and interpretable predictive model for disease prediction in comparison to deep learning models (primarily $[6,7]$ ) which are currently the focus of research work in disease prediction domain. The focus of this work was to push the capabilities of a tree based model and come up with a strong baseline. It was surprising to observe that our fine-tuned model outperformed these deep learning solutions by a good margin. This was despite the fact that both deep learning implementations were fine-tuned with respect to the same datasets. We also presented strategies to interpret our model at both global and instance levels. The instance level interpretation utilised SHAP framework[9]. We also presented a brief discussion on the effectiveness of tree based approaches for our task. 


\section{Acknowledgements}

We would like to thank Vineet Shukla \& Saikumar Chintareddy and the diagnosis prediction team for their contributions along with Dr. Manotosh Das \& Dr. Bidisha Ghoshal for helping us with their medical expertise.

\section{References}

[1] G Maragatham and S. Devi. "LSTM model for prediction of heart failure in big data". In: Journal of medical systems 43.5 (2019), pp. 1-13.

[2] A. N. Jagannatha and H. Yu. "Bidirectional RNN for Medical Event Detection in Electronic Health Records". In: Proceedings of the conference. Association for Computational Linguistics. North American Chapter. Meeting 2016 (2016), pp. 473-482.

[3] D. Bahdanau, K. Cho, and Y. Bengio. "Neural Machine Translation by Jointly Learning to Align and Translate". In: arXiv e-prints (Sept. 2014), arXiv:1409.0473. arXiv: 1409.0473 [cs.CL].

[4] E. Choi, M. T. Bahadori, A. Schuetz, W. F. Stewart, and J. Sun. "Doctor AI: Predicting Clinical Events via Recurrent Neural Networks". In: JMLR workshop and conference proceedings 56 (2015), pp. 301-318.

[5] Y. Zhang. "ATTAIN: Attention-based Time-Aware LSTM Networks for Disease Progression Modeling." In: In Proceedings of the 28th International Joint Conference on Artificial Intelligence (IJCAI-2019), pp. 4369-4375, Macao, China. 2019.

[6] E. Choi, M. T. Bahadori, J. Sun, J. Kulas, A. Schuetz, and W. Stewart. "RETAIN: An Interpretable Predictive Model for Healthcare using Reverse Time Attention Mechanism". In: Advances in Neural Information Processing Systems. Ed. by D. Lee, M. Sugiyama, U. Luxburg, I. Guyon, and R. Garnett. Vol. 29. Curran Associates, Inc., 2016, pp. 3504-3512.

[7] F. Ma, R. Chitta, J. Zhou, Q. You, T. Sun, and J. Gao. "Dipole: Diagnosis Prediction in Healthcare via Attention-Based Bidirectional Recurrent Neural Networks". In: Proceedings of the 23rd ACM SIGKDD International Conference on Knowledge Discovery and Data Mining. KDD '17. Halifax, NS, Canada: Association for Computing Machinery, 2017, pp. 1903-1911. ISBN: 9781450348874. DOI: 10.1145/3097983.3098088.

[8] C. Xiao, E. Choi, and J. Sun. "Opportunities and challenges in developing deep learning models using electronic health records data: a systematic review". In: Journal of the American Medical Informatics Association 25.10 (2018), pp. 1419-1428.

[9] S. M. Lundberg and S.-I. Lee. "A Unified Approach to Interpreting Model Predictions". In: Advances in Neural Information Processing Systems 30. Ed. by I. Guyon, U. V. Luxburg, S. Bengio, H. Wallach, R. Fergus, S. Vishwanathan, and R. Garnett. Curran Associates, Inc., 2017, pp. 4765-4774.

[10] T. Chen and C. Guestrin. "XGBoost". In: Proceedings of the 22nd ACM SIGKDD International Conference on Knowledge Discovery and Data Mining (Aug. 2016). DoI: 10.1145/ 2939672.2939785.

[11] J. Davis and M. Goadrich. "The Relationship between Precision-Recall and ROC Curves". In: Proceedings of the 23rd International Conference on Machine Learning. ICML 06. Pittsburgh, Pennsylvania, USA: Association for Computing Machinery, 2006, pp. 233-240. ISBN: 1595933832. DOI: 10.1145/1143844.1143874.

[12] XGBoost Python Package. URL: https : / /xgboost.readthedocs.io/en/latest/python/ python_api.html.

[13] shivamb. Data Science Trends on Kaggle !! 2018. uRL: https://www.kaggle.com/shivamb/ data-science-trends-on-kaggle.

[14] A. Criminisi, J. Shotton, and E. Konukoglu. Decision Forests: A Unified Framework for Classification, Regression, Density Estimation, Manifold Learning and Semi-Supervised Learning. Foundations and Trendső in Computer Graphics and Vision: Vol. 7: No 2-3, pp 81-227. Vol. 7. 2-3. NOW Publishers, Jan. 2012, pp. 81-227.

[15] D. H. Wolpert and W. G. Macready. "No free lunch theorems for optimization". In: IEEE transactions on evolutionary computation 1.1 (1997), pp. 67-82. 